\title{
CUSTOS HOSPITALARES DAS CEFALÉIAS AGUDAS EM UMA UNIDADE DE EMERGÊNCIA PÚBLICA BRASILEIRA
}

\author{
MARCELO E. BIGAL*, LUCIANA C. FERNANDES ${ }^{* *}$ CARLOS A. BORDIN/**, JOSÉ G. SPECIALI***
}

\begin{abstract}
RESUMO - Cefaléia é uma das queixas mais freqüentes na prática médica e causa bastante comum de atendimento em unidades de emergência, implicando custos consideravelmente altos. Foram estudados retrospectivamente os casos de cefaléia atendidos na Unidade de Emergência do Hospital das Clinicas de Ribeirão Preto (UE) no ano de 1996. Neste ano, 1254 pacientes procuraram a UE com queixa de cefaléia aguda, sendo que 64 necessitaram de internação. O custo estimado com o atendimento, investigação e tratamento clínico das cefaléias agudas foi da ordem de R \$ 138,573.31 (US\$ 76,985.17). Os gastos apenas com exames laboratoriais foram R $\$ 23,801.54$ (US\$13,223.07). Os custos cirúrgicos foram R \$ 5,817.90 (US\$3,232.17). Os custos totais foram RS\$144,391.21 (US\$ 80,217.34), o que equivale a R $\$ 115,14$ (US\$ 63.97) por paciente. Tais cálculos estimulam discussão adicional acerca de custos e eficácia do modelo de saúde vigente, em que os recursos financeiros são precários e as necessidades da população dependente do estado são proporcionalmente maiores.
\end{abstract}

PALAVRAS-CHAVE: cefaléia, unidade de emergência, custos.

\section{Hospitalar costs of acute headaches in a Brazilian public emergency room unit}

ABSTRACT - Headache is one of the most frequent complain in the medical practice and a very common cause of medical assistance searching in emergency rooms, leading to considerable high costs. The headache cases assisted during the year of 1996 of an emergency room unit (UE - USP) at Ribeirão Preto, SP, Brazil, had been retrospectively studied. During that year a number of 1254 patients searched the UE - USP with major complain of acute headache, among which 64 needed hospitalization. The estimated costs due to consultation, investigation and clinical treatment of the acute headaches was in the order of R $\$ 138573.31$ (US\$ 76 985.17). The expenses related only to laboratorial exams were R $\$ 23801.54$ (US\$ 13 223.07). The surgical expenses were R 5817.90 (US\$ 3232.17 ). The total cost was R $\$ 144391.21$ (US\$ 80217.34 ) which corresponds to R $\$ 115.14$ (US\$ 63.97) per patient. This calculus instigates an additional discussion about the costs and effectiveness of the current public health policy, where the financial resources are less abundant than the State dependant population's needs.

KEY WORDS: headache, emergency room unit, costs.

Desde os primórdios da civilização o homem tem se preocupado com o sintoma cefaléia, com as formas de evitá-lo e de eliminá-lo. Sua importância, já ressaltada em textos assírios e mesopotâmicos, foi definitivamente assinalada na literatura há 2500 anos quando Hipócrates, descreveu o primeiro caso de migrânea. A cefaléia é um dos sintomas mais comuns na clínica médica havendo estimativas de que $90 \%$ dos homens e $95 \%$ das mulheres tenham cefaléias anualmente ${ }^{1}$. Pode estar associada a diversas condições, como trauma de crânio, intoxicação alcoólica, depressão, tumor ou representar uma cefaléia primária como a migrânea. Acarreta impacto considerável na qualidade de vida de seus portadores ${ }^{2-3}$. Estudos consideram a migrânea mais incapacitante que doenças como hipertensão arterial, osteoartrite e diabetes ${ }^{4}$. Além desse impacto negativo no bem-estar individual, há ainda prejuízo econômico acarretado, que repercute sobre o

Faculdade de Medicina de Ribeirão Preto (FMRP) da Universidade de São Paulo (USP): *Mestre em Neurologia pela FMRP-USP; **Psicóloga, pós-graduanda em neurosciências pela FMRP-USP; ***Doutor em Neurologia pela FMRP-USP; ****Professor Associado de Neurologia da FMRP-USP. Aceite: 15-abril-2000.

Dr. José G. Speciali - Faculdade de Medicina de Ribeirão Preto, Departamento de Neurologia Av. Bandeirantes 3900 - 14049-900 Ribeirão Preto, SP - Brasil. 
próprio indivíduo e sobre a sociedade ${ }^{5}$. A mensuração desse prejuízo envolve cálculos dos custos diretos (gastos com o sistema de saúde - atenção médica, exames e medicamentos) ${ }^{6}$, custos indiretos (prejuízos pelas faltas ao trabalho e diminuição da produtividade) ${ }^{7}$ e custos agregados (pesquisas, mobilização de material e pessoal indiretamente envolvido com a doença $)^{8}$.

Por serem altamente prevalentes, as cefaléias acarretam impacto significativo no sistema de saúde, seja ele público ou privado. Em nosso meio, um estudo realizado em unidades básicas de saúde mostrou que $9,3 \%$ das consultas em adultos eram secundárias às mesmas ${ }^{9}$. Nos EUA, estimase que $40 \%$ da população apresente cefaléia suficientemente intensa, em algum momento de suas vidas, que os leve a procurar assistência médica ${ }^{10}$. Nesse país o custo direto anual, apenas da migrânea, foi calculado em US\$ 9,6 bilhões ${ }^{11}$.

O presente estudo tem por objetivo estimar os custos do atendimento hospitalar, em uma Unidade de Emergência (UE), de pacientes com cefaléia aguda.

\section{MÉTODO}

Foram estudados retrospectivamente os prontuários dos pacientes atendidos na Unidade de Emergência do Hospital das Clínicas da Faculdade de Medicina de Ribeirão Preto - USP (HC-FMRP-USP), no ano de 1996 (1 de janeiro de 1996 a 31 de dezembro de 1996), com queixa principal de cefaléia. Foram excluídos os prontuários de pacientes cujo problema principal foi trauma crânio-encefálico agudo, pacientes politraumatizados e pacientes que, embora queixando-se de cefaléia, essa não fosse apresentada como queixa principal.

Para o cálculo dos custos hospitalares gerados pelo atendimento de cefaléia na UE, utilizamos o "Anuário Estatístico do HC-FMRP-USP" ${ }^{12}$, que apresenta os dados estatísticos referentes aos atendimentos realizados no referido ano. Utilizamos ainda informações oficiais colhidas diretamente, e que serão publicadas no próximo ano (anuário 1999) na Sessão de Custos do HC-FMRP-USP. Parte desses dados diz respeito à planilha de gastos da instituição, incluindo os gastos devido aos atendimentos, ambulatoriais e de urgência. Com o objetivo de obtermos um panorama de gastos atualizado, ao invés de utilizarmos os valores monetários do ano de 1996, trabalhamos com valores vigentes no primeiro trimestre do ano de 1999 e utilizamos a seguinte metodologia:

1 - Custos do Atendimento Médico: tais custos englobam os gastos em atendimento, exames laboratoriais (calculados também em separado, conforme detalhado no item 2 ) e tratamento clínico, excluindo os gastos com tratamento cirúrgico. Foram obtidos tomando por base os seguintes valores:

1.1 - Custos do Atendimento Médico Ambulatorial: são os custos dos pacientes que permaneceram menos de 12 horas (não internados) na UE. Para os pacientes com menos de 12 anos o custo foi, no primeiro trimestre do ano de 1999, da ordem de R \$ 164,69 por atendimento. Para os pacientes com 12 anos ou mais o custo foi de R\$ 78,45. Multiplicamos então o número de pacientes com cada uma dessas faixas etárias pelo valor correspondente.

1.2 - Custos com Internação: tais custos foram, para pacientes com menos de 12 anos, $\mathrm{R} \$ 226,54$ / dia e para pacientes com 12 anos ou mais, $\mathrm{R} \$ 173,58$ / dia.

2 - Custos com Exames Laboratoriais: para a avaliação dos gastos com exames laboratoriais, multiplicou-se cada exame pelo seu respectivo valor. O valor de cada exame é obtido, segundo a metodologia utilizada pelo HC, dividindo o valor total envolvido para a manutenção e funcionamento do laboratório em questão em determinado período, pelo número de exames solicitados nesse mesmo período. Assim, por exemplo para o laboratório de líquido cefaloraqueano os gastos com esse laboratório envolvem os salários dos funcionários, materiais para os exames, gastos com luz, água, limpeza e demais gastos. Dividindo-se o valor assim obtido pelo número de exames solicitados, chega-se ao valor unitário do exame. Para os pacientes internados, todos os exames foram avaliados. Para os pacientes não internados, os percentuais dos exames solicitados em uma amostra avaliada (197 pacientes, escolhidos aleatoriamente) foram extrapolados para os demais pacientes não internados. Com tal metodologia determinamos quanto dos gastos totais para os pacientes com cefaléia foram devido aos exames laboratoriais.

3 - Custos Cirúrgicos: o custo cirúrgico no HC é dividido em 6 categorias, que são formadas levando-se em conta o porte cirúrgico e o porte anestésico de cada procedimento. Multiplicamos cada uma das cirurgias realizadas em pacientes que deram entrada na UE com queixa principal de cefaléia pelo respetivo custo cirúrgico.

4 - Custos Totais: obtidos da soma dos custos do atendimento médico com os custos cirúrgicos.

Os valores em dólares foram calculados de acordo com o câmbio comercial de 15 de julho de 1999. 
Tabela 1. Custo unitário e custo total dos exames laboratoriais solicitados para os pacientes do grupo NI.

\begin{tabular}{lccc}
\hline Exames laboratoriais & $\begin{array}{c}\text { Total de exames } \\
\mathrm{n}\end{array}$ & $\begin{array}{c}\text { Custo unitário } \\
\mathrm{R} \$ *\end{array}$ & $\begin{array}{c}\text { Custo Total } \\
\mathrm{R} \$\end{array}$ \\
\hline Exames de sangue** & 207 & 2,61 & 540,27 \\
Radiografias de crânio / face & 162 & 12,27 & $1.987,74$ \\
TC crânio & 81 & 128,62 & $10.418,22$ \\
Punção / LCR & 37 & 45,94 & $1.699,78$ \\
EEG & 19 & 156,44 & $2.972,36$ \\
Total & & & $17.618,37$ \\
\hline
\end{tabular}

TC, tomografia computadorizada; LCR, líquor; EEG, eletrencefalograma. *Valores vigentes no primeiro trimestre de 1999. **Cada coleta de exame de sangue em paciente NI originou 3 exames em média.

Tabela 2. Custo unitário e custo total dos exames laboratoriais solicitados para os pacientes do grupo I.

\begin{tabular}{cccc}
\hline Exames laboratoriais & $\begin{array}{c}\text { Total de exames } \\
\mathrm{n}(\%)\end{array}$ & $\begin{array}{c}\text { Custo unitário } \\
\text { (reais)* }\end{array}$ & $\begin{array}{c}\text { Custo total } \\
\text { (reais) }\end{array}$ \\
\hline Exames de sangue** & 112 & 2,61 & 292,32 \\
Radiografias de crânio / face & 18 & 12,27 & 220,86 \\
TC crânio & 26 & 128,62 & $3.344,12$ \\
Punção/LCR & 21 & 45,94 & 964,74 \\
RNM & 3 & 309,73 & 929,19 \\
Angiografia & 1 & 193,70 & 193,70 \\
US de carótidas & 1 & 81,80 & 81,80 \\
EEG & 1 & 156,44 & 156,44 \\
Valor total & & & $6.183,17$ \\
\hline
\end{tabular}

TC, tomografia computadorizada; LCR, líquor; RNM, ressonância nuclear magnética; US, ultrassom; EEG, eletroencefalograma. *Valores vigentes no primeiro trimestre de 1999. **Cada coleta de exame de sangue em paciente I originou 4 exames em média.

\section{RESULTADOS}

Um total de 1254 pacientes deram entrada na UE com queixa principal de cefaléia, no ano de 1996. Desses, 64 (5,1\%) necessitaram de internação (permaneceram mais de 12 horas em ambiente hospitalar). Os dados epidemiológicos e referentes ao diagnóstico e tratamento já foram publicados ${ }^{13}$. Dentre os pacientes não internados (NI), cefaléias primárias foram diagnosticadas em $77 \%$, cefaléias secundárias a distúrbios neurológicos em $9,1 \%$ e cefaléia secundária a distúrbios sistêmicos em $13,9 \%$. Nos pacientes internados ( I ) esses valores foram, respectivamente, $29,7 \% ; 51,5 \%$ e $18,8 \%$.

\section{1 - Custos dos exames laboratoriais}

As tabelas 1 e 2 apresentam respectivamente os gastos com os exames laboratoriais solicitados para investigação respectivamente dos pacientes NI e I. Os valores dos exames foram calculados tomando por base o primeiro trimestre do ano de 1999.

O total de gastos em exames laboratoriais para pacientes com cefaléia foi R \$23.801,54 (US\$ 13,223.07). Considerando os 1254 pacientes, gastou-se, com exames laboratoriais, em média, R\$ 18,98 (US\$ 10.54) por paciente. 
É importante salientar que os padrões de solicitação dos exames laboratoriais não se alteraram entre os anos de 1996 e 1999. Assim, por trabalharmos com valores corrigidos para o ano de 1999, de dados obtidos no ano de 1996, obtivemos panorama atualizado dos custos de exames laboratoriais, sem incorrer em viés de informação,

\section{2 - Custos com atendimento médico}

Os custos que serão apresentados a seguir compreendem os gastos com atendimento, investigação laboratorial e tratamento clínico. Englobam, portanto, os custos com exames laboratoriais, que foram detalhados nas tabelas 1 e 2, mas excluem os gastos com procedimentos cirúrgicos, que serão apresentados no item seguinte.

\section{1 - Custos com atendimento médico ambulatorial (pacientes não internados)}

Dos 1190 pacientes que não necessitaram internação, 131 apresentavam idade menor que 12 anos. $\mathrm{O}$ atendimento ambulatorial (atendimento dos pacientes que não necessitaram internação) custou, no primeiro trimestre do ano de 1999, em média R \$ 164,69 (US\$ 91.49) por paciente nessa faixa etária. Os gastos com esses atendimentos foram, portanto, da ordem de R $\$ 21.574,39$ (US\$ 11,985.77).

Os 1059 pacientes restantes apresentavam mais de 12 anos, sendo que o atendimento ambulatorial desses casos custou, no mesmo período, R $\$ 78,45$ (US\$ 43.65). Os gastos com esses atendimentos foram, portanto, da ordem de $\mathrm{R} \$ 83.078,55$ (US\$ 46,154.75).

O total de gastos com os pacientes não internados foi estimado, desta maneira, em R\$ 104.652,94 (US\$ 58,140.22).

\section{2 - Custos com atendimento médico dos pacientes internados}

Dos 64 pacientes que necessitaram internação, 17 apresentavam menos de 12 anos e ficaram em média 3,3 dias internados, sendo responsáveis, portanto, por 56,1 diárias. Cada diária para pacientes desta faixa etária custou, no primeiro trimestre do ano de 1999, R \$ 226,54 (US\$ 125.86). Assim, o custo estimado foi de R \$ 12.708,89 (US\$ 7,060.49).

Os 47 pacientes com 12 anos ou mais ficaram, em média, 2,6 dias internados, sendo responsáveis por 122,2 diárias, a um valor unitário, de R $\$ 173,58$ (US\$ 96.43), o que corresponde a um custo estimado em $\mathrm{R} \$ 21.211,48$ (US\$ 11,784.15).

O total estimado dos gastos com os pacientes internados foi, portanto, R\$33.920,37 (US\$ $18,844.65)$.

\section{3 - Custos totais com atendimento médico (clínico)}

Dos cálculos acima estima-se, portanto, que em valores atuais, o $\mathrm{HC}$ despendeu recursos da ordem de R \$ 138.573,31 (US\$ 76,985.17) com o atendimento, investigação laboratorial e tratamento clínico das cefaléias agudas no ano de 1996. Considerando os 1254 pacientes que procuraram a UE no ano de 1996 com cefaléia, gasta-se, para este fim, em média R \$110,50 (US\$ 61.39) por paciente.

\section{3 - Custos Cirúrgicos}

Realizou-se um total de 11 cirurgias em pacientes que procuraram ou foram trazidos a UE com queixa principal de cefaléia, todas em pacientes que necessitaram internação (grupo I).

A divisão de finanças do HC - FMRP - USP trabalha com 6 categorias de custos cirúrgicos, calculados de acordo com o porte cirúrgico e anestésico. A tabela 3 apresenta o cálculo de custos das 11 cirurgias realizadas no ano de 1996, tomando por base os valores do primeiro trimestre do ano de 1999 .

\section{4 - Custos Totais}

Somando-se os custos do atendimento médico (item 2), com os custos cirúrgicos (item 3), concluímos que, trabalhando com valores atuais, os gastos com pacientes com cefaléia seriam da 
Tabela 3. Custos cirúrgicos em pacientes que deram entrada na UE com queixa principal de cefaléia.

\begin{tabular}{cccc}
\hline Porte cirúrgico & Número de pacientes & $\begin{array}{c}\text { Custo Unitário* } \\
\text { R \$ (US\$) }\end{array}$ & $\begin{array}{c}\text { Custo Total } \\
\text { R \$ (US\$) }\end{array}$ \\
\hline 1 & 2 & $141,90(78.83)$ & $283,80(157.67)$ \\
2 & 2 & $283,80(157.67)$ & $567,60(315.33)$ \\
3 & 1 & $425,70(251.50)$ & $425,70(251.50)$ \\
4 & 4 & $567,60(315.33)$ & $2.270,40(1261.33)$ \\
5 & 1 & $709,50(394.17)$ & $709,50(394.17)$ \\
6 & 1 & $1560,90(867.17)$ & $1.560,90(867.17)$ \\
Total & & & $5.817,90(3.232 .17)$ \\
\hline
\end{tabular}

*Valores vigentes no primeiro trimestre de 1999. Dólar cotado em 15 de julho de 1998.

ordem de R \$ 144.391,21 (US\$ 80,217.34). Considerando os 1254 pacientes, o custo total per capita foi de R \$ 115,14 (US\$ 63.97).

Se subtrairmos, dos gastos totais, os gastos com exames laboratoriais, internações hospitalares e cirurgias, obteremos os gastos com atendimento médico ambulatorial simples (aquele em que o paciente ficou menos de 12 horas, ou seja, não necessitou internação ou cirurgia e para o qual não se pediu nenhum exame laboratorial). Esses foram da ordem de R \$ 80.851,14 (US\$ 44,917.30) e responderam pela maior parte dos custos estimados totais, o que pode ser observado na Figura 1.

\section{DISCUSSÃO}

A alta prevalência das cefaléias na população e o impacto por elas acarretado repercutem nas unidades de emergência. Trabalhos avaliam que a cefaléia seria responsável por $1 \%$ a $16 \%$ de todas as visitas nessas unidades ${ }^{14-17}$. A discrepância dos dados acima se deve à diferença de metodologia

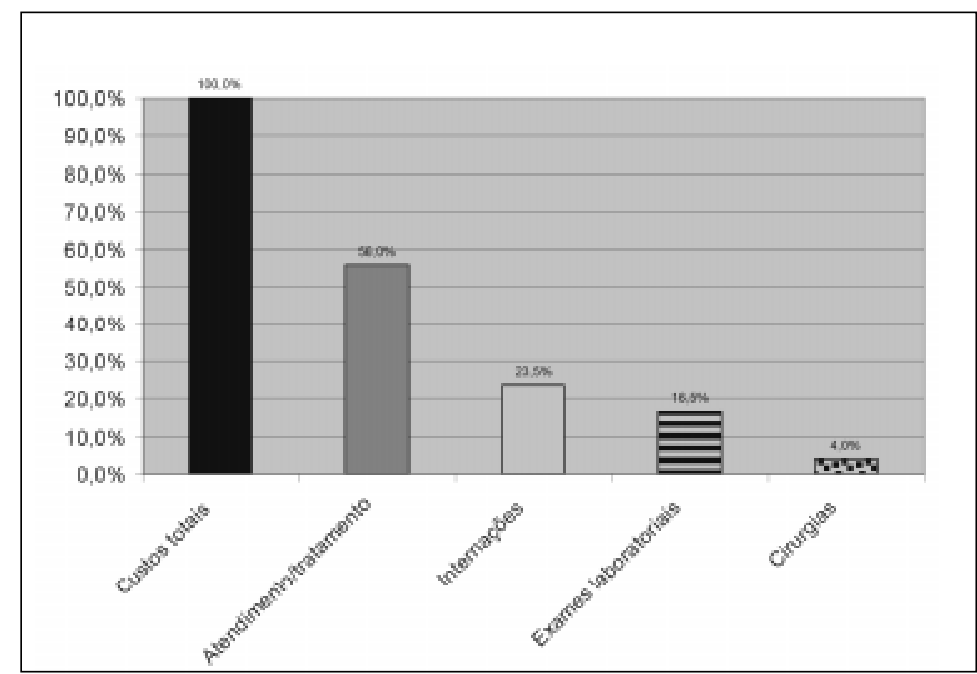

Fig 1. Percentuais dos determinantes dos custos estimados totais para o atendimento, diagnóstico e tratamento da cefaléia na UE, ano de 1996. 
utilizada: alguns foram feitos em unidades gerais, outros em unidades de atendimento apenas clínico, alguns excluíram traumatismos cranianos e suas complicações, enquanto outros não. Carvalho ${ }^{18} \mathrm{em}$ estudo realizado em unidade de atendimento de emergências neurológicas, refere cefaléia como sendo responsável por $56 \%$ de todos os atendimentos. A maioria dos estudos defende, no entanto, que cefaléia seria sintoma responsável por $1 \%$ a $3 \%$ de todas as consultas em unidades de emergência ${ }^{19,20}$. Silberstein e Silberstein consideram o valor de $1 \%$ como o mais extrapolável para diferentes unidades ${ }^{21}$.

O custo das cefaléias é consideravelmente alto, tanto para os pacientes como para a sociedade. Estima-se que, nos EUA, apenas no que diz respeito à perda da produtividade no trabalho, a migrânea cause prejuízos anuais que podem chegar aos 17,2 bilhões de dólares ${ }^{8}$. Vincent et al, em estudo realizado em uma empresa no Brasil, estimaram os custos indiretos das cefaléias em $\mathrm{R} \$ 144.682,39$ por ano ${ }^{22}$. Os custos diretos das cefaléias também são consideráveis. Celentano et al. referem que $13,4 \%$ dos migranosos procuram unidades de emergência ao menos uma vez devido à sua dor ${ }^{23}$. Apenas com hospitalizações, gasta-se, no Reino Unido, o equivalente a 2,19 milhões de libras esterlinas por $\mathrm{ano}^{24}$.

Unidades de emergência são bastante onerosas para o Estado, dada sua complexidade e natureza do atendimento oferecido. Em um ano a UE gastou, em valores atualizados, o equivalente a R\$144.391,21 ou R \$115,14 por paciente atendido. Considerando-se o câmbio oficial, gastou-se, portanto, o equivalente a US\$ 63.97/paciente. Os pacientes NI custaram o equivalente a R \$ 87,94/paciente (US\$ 48.85) e os pacientes I, considerando o custo cirúrgico, o equivalente a R \$ 1150,91/paciente (US\$ 639.39). São poucos os trabalhos disponíveis sobre custos de cefaléia em UE. Osterhaus et al. ${ }^{25}$, trabalhando apenas com a migrânea, encontraram valores de atendimento superiores aos nossos: 1) visitas a UE: custos de US\$ 281.00 / paciente; 2) hospitalizações: custo de US\$ 387.00 / paciente. Os custos de nossas hospitalizações, desconsideradas as cirurgias, foram de R \$ 530,00 / paciente (US\$294.44).

Alguns dados referentes aos custos hospitalares de pacientes com cefaléia aguda na UE chamam a atenção: 1 - Os gastos com exames laboratoriais dos pacientes NI representam 16,5\% do total; 2 Os custos com internações (incluindo os exames laboratoriais dos pacientes internados) representaram 23,5\% do total; 3 - Os custos cirúrgicos representaram 4\% do total dos gastos. Assim, $56 \%$ dos gastos com atendimento, diagnóstico e tratamento das cefaléias na UE (R \$ 80.859,07) deram-se com pacientes que permaneceram menos de 12 horas em ambiente hospitalar, não necessitaram exames laboratoriais, internações ou cirurgias. A grande maioria dos mesmos apresentava cefaléia primária, cujo diagnóstico é eminentemente clínico e o tratamento instituído consistiu de medicamentos existentes em unidades de menor complexidade. O custo da má resolutividade do sistema público de saúde no tocante a esse sintoma é bastante elevado.

Há que se tecer um comentário à metodologia que o Hospital das Clínicas de Ribeirão Preto se utiliza no cálculo dos custos dos exames laboratoriais. Ao se dividir toda a verba empregada em um laboratório pelo número de exames laboratoriais realizados no mesmo, há uma diminuição do valor do exame diretamente proporcional ao número de exames realizados. Isso gera algumas distorções, como por exemplo um eletroencefalograma (o laboratório de neurofisiologia da UE realiza poucos exames) custar mais que uma tomografia computadorizada (o laboratório de neuroimagem realiza muitos exames). No entanto consideramos tal metodologia correta, por refletir adequadamente a maior produtividade de determinados setores. Calcular de outro modo (considerando apenas a complexidade do exame ou o previsto em tabelas de ressarcimento) seria falsear os custos reais.

É importante ressaltar que o paciente que chega a UE vem encaminhado de outra unidade (UBS), onde foi consultado previamente. Há, portanto, o custo resultante dessa consulta. Assim o paciente encaminhado desnecessariamente à UE acarreta custo duplo ao Estado, o do atendimento na UBS e o do próprio atendimento na UE. Além disso, o encaminhamento de pacientes com patologias potencialmente manejáveis a nível primário para uma unidade terciária implica em: 1) Mobilização de transporte em veículo especial (ambulância), que necessita também da disponibilização de pessoal especializado; 2) Agravamento da falta de leitos disponíveis para a utilização em casos mais sérios; 3) 
Demora adicional para se resolver o problema do paciente, com conseqüente prolongamento do sofrimento do mesmo e de absenteísmo no trabalho. Ou seja, além do acréscimo nos custos diretos há também aumento adicional dos custos indiretos e dos custos operacionais.

Tais cálculos estimulam discussão adicional acerca de custos e eficácia do modelo de saúde em questão, temas muito debatidos em países desenvolvidos e que deveriam sê-lo de maneira contumaz também nos países em desenvolvimento, onde os recursos financeiros disponíveis são menos abundantes, e as necessidades da população dependente do estado são maiores.

\section{REFERÊNCIAS}

1. Rasmussen BK, Jansen R, Olesen J. A population-based analysis of the diagnostic criteria of the International Headache Society. Cephalalgia 1991;11:129-134.

2. Osterhaus JT. The burden of migraine. Can J Neurol Sci 1993;20:35-39.

3. Dahlöf C. Assessment of health-related quality of life in migraine. Cephalalgia 1993;13:233-237.

4. Solomon GD, Skobieranda FG, Gragg LA. Quality of life and well-being of headache patients: measurements by the Medical Outcome Study Instrument. Headache 1993;33:351-358.

5. Lipton RB, Stewart WF, Von Korff M. The burden of migraine. Pharmacoeconomics 1994;6:215-221.

6. Clouse JC, Osterhaus, JT. Healthcare resource use and costs associated with migraine in a managed healthcare setting. Ann Pharmacother 1994;28:659-664.

7. Rasmussen BK, Jensen R, Olesen J. Impact of headache on sickness absence and utilisation of medical services: a Danish population study. J Epidemiol Community Health 1992;46:443-446.

8. Stang PE, Osterhaus JT. Impact of migraine in the United States: data from the national Health Interview Survey. Headache 1993;33:29-35.

9. Bigal ME, Bordini CA, Speciali JG. Etiology and distribution of headaches in two Brazilian primary care units. Headache 1999 (in press).

10. Speciali JG. Simpósio cefaléia: introdução. Medicina Ribeirão Preto 1997;30:419-420.

11. De Lissovoy, Lazarus SS. The economic cost of migraine: present state of knowledge. Neurology 1994;44(Suppl 4):56-62.

12. Anuário Estatístico do Hospital das Clínicas da Faculdade de Medicina de Ribeirão Preto - USP. Ribeirão Preto: Superintendência do HC - FMRP - USP, 1996.

13. Bigal ME, Bordini CA, Speciali JG. Tratamento da cefaléia em uma unidade de emergência da cidade de Ribeirão Preto. Arq Neuropsiquiatr 1999;57:813-819.

14. Dhopesh V, Amwar R, Herring G. A retrospective assessment of emergency department pacients with complaint of headache. Headache 1979;19:37-42.

15. Dickman RL, Masten T. The management of non-traumatic headache in an university hospital emergency room. Headache 1979;19:391-396.

16. Leight MJ. Non-traumatic headache in the emergency department. A J Emerg Med 1980;9:404-409.

17. Luda E, Comitangelo R, Sicura L. The symptom of headache in emergency departments: the experience of a neurology emergency department. Ital J Neurol Sci 1995;16:259-301.

18. Carvalho JJF. Cefaléia na unidade de emergência. São Paulo, 1999: Anais do XVIII Congresso Brasileiro de Neurologia.

19. Olenick JS, Taylor RB. Emergency evaluation and treatment of headache. Primary Care 1986;13:97-107.

20. Barton CW. Evaluation and treatment of headache in the emergency department: a survey. Headache 1994;34:91-94.

21. Silberstein SD, Silberstein MM. New concepts in the pathogenesis of migraine headache. Pain Management 1990;3:297-302.

22. Vincent MB, Rodrigues AJ, Oliveira GV, et al. Prevalência e custos indiretos das cefaléias em uma empresa brasileira. Arq Neuropsiquiatr 1998;56:734-743.

23. Celentano DD, Stewart WF, Lipton RB, et al. Medication use and disability among migrainours:a national probability sample survey. Headache 1992;32:223-228.

24. Blau JN, Drummond MF. Office of health economics: migraine. London, 1991;204-218.

25. Osterhaus JT, Guterman DL, Plachetcka JR. Healthcare resource and lost labour costs of migraine headache in the US. PharmacoEconomics 1992;2:67-76. 